\title{
A pragmatic approach to monitoring the continuum of HIV care in the absence of a HIV register
}

\section{Hurley $\mathrm{C}^{1}$, O’Donnell $\mathrm{K}^{2}$, De Gascun $\mathrm{C}^{3}$, Cooney $\mathrm{F}^{4}$, Butler $\mathrm{K}^{5}$, Lynam $\mathrm{A}^{6}$, Tuite $\mathrm{H}^{7}$, Igoe $\mathrm{D}^{2}$, Lyons $\mathrm{F}^{1}$ on behalf of the Continuum of HIV Care Steering Group}

${ }^{1} \mathrm{HSE}$ Sexual Health \& Crisis Pregnancy Programme (SHCPP); ${ }^{2}$ Health Protection Surveillance Centre (HPSC); ${ }^{3} \mathrm{UCD}$ National Virus Reference Laboratory; ${ }^{4}$ Department of Public Health, HSE-East; ${ }^{5}$ Our Lady's Children's Hospital Crumlin; ${ }^{6}$ GUIDE Clinic, St. James's Hospital; 7 University Hospital Galway

\section{Background}

Antiretroviral therapy (ART) is recommended for all people living with HIV (PLHIV) in Ireland ${ }^{1}$, in line with international guidelines. Ireland does not currently have a national HIV outcomes register and therefore this work was carried out to ascertain degree of compliance with these guidelines at national level.

The continuum of HIV care is a conceptual framework that enables countries to monitor the effectiveness of key areas of their HIV programme. The European Centre for Disease Prevention and Control (ECDC) recommends a standardised approach and has developed definitions for the four priority stages of the continuum of HIV care, in line with the UNAIDS 90-90-90 targets.

\section{Aim \& Methods}

Aim: to develop a pragmatic and consistent approach to monitoring the continuum of HIV care in Ireland.

A Steering Group was established with clinical, laboratory, public health, and surveillance representation. The ECDC definitions were adapted for Ireland and agreed by the Steering Group.

The HPSC worked with UNAIDS to develop Spectrum modelling estimates for the first two stages of the continuum. A nationally coordinated audit of all HIV treatment services was conducted to provide data for the third and fourth stages of the continuum. In line with the agreed definitions, some services collected their own data and a representative from the SHCPP assisted some services in compiling their data. The audit results were collated by the SHCPP.

\section{Results}

Modelling estimated that 7,205 (95\% Cl: 6,456-8,056) people are living with HIV in Ireland (stage 1 ). An estimated $12.9 \%$ of these are undiagnosed and an estimated 6,276 (95\% Cl:

$5,623-7,017)$ have been diagnosed (stage 2).

\begin{tabular}{|l|c|}
\hline Spectrum figures & Estimate $(95 \%$ Cl) \\
\hline Population in Ireland living with HIV & $7,205(6,456-8,056)$ \\
\hline Proportion living with undiagnosed HIV & $12.9 \%$ \\
\hline Number living with undiagnosed HIV & $929(833-1,039)$ \\
\hline Number diagnosed with HIV & $6,276(5,623-7,017)$ \\
\hline
\end{tabular}

Table 1. UNAIDS Spectrum estimates for stages 1 and 2 , Ireland 2017

\section{Results}

The clinical audit found that 5,317 individuals attended a HIV treatment service in Ireland in 2017 (Figure 1). Of these, 98.3\% were on ART, of whom $95.4 \%$ were virally supressed to $<200 \mathrm{cpm}$ and $90.6 \%$ were virally suppressed to below the level of detection of the assay.

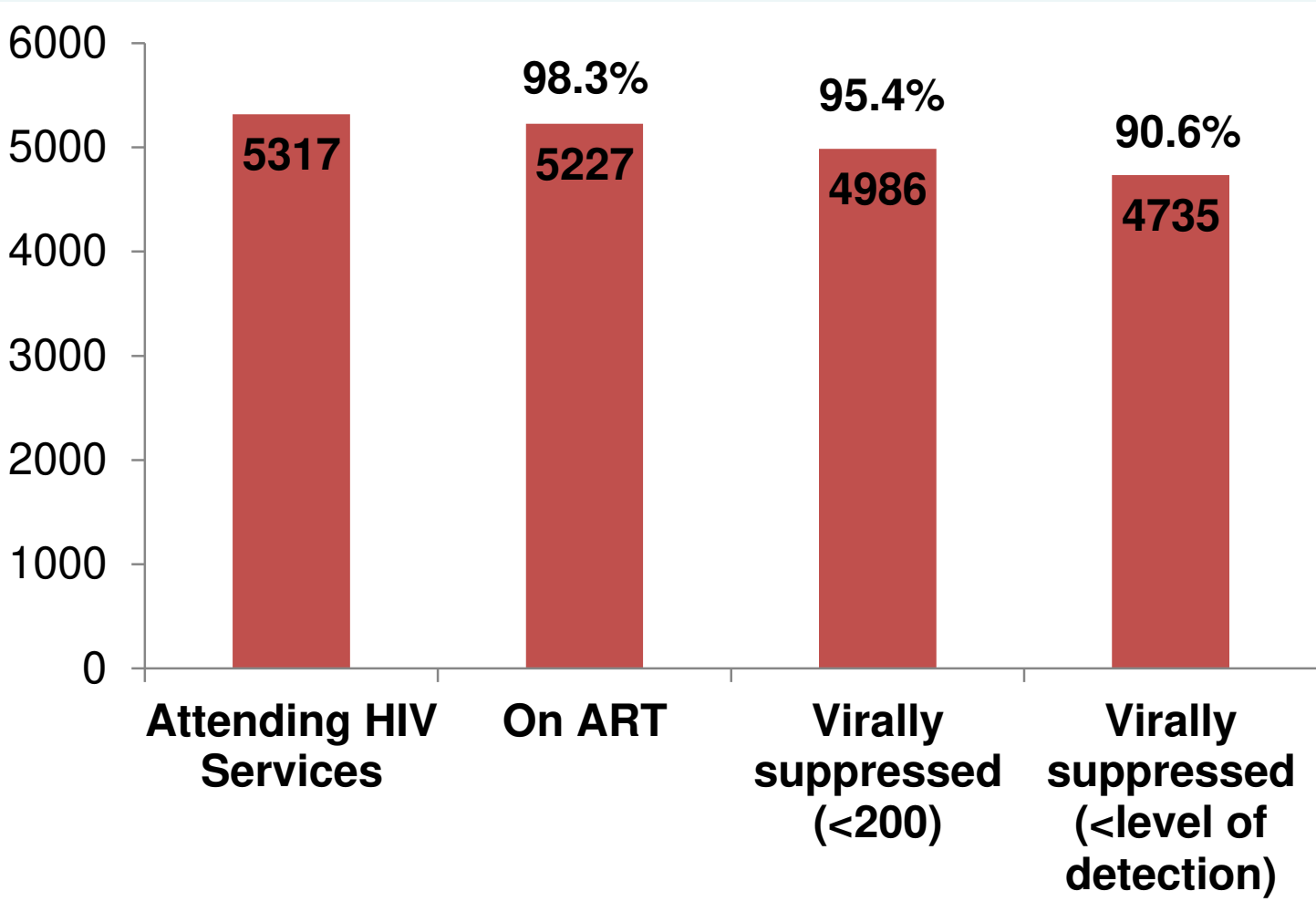

Figure 1. HIV treatment audit, 2017

Combining the modelling estimates with the audit of HIV treatment (Figure 2 ), it is estimated that $87.1 \%$ of PLHIV are diagnosed, $83.3 \%$ of these are on ART, of whom $95.4 \%$ are virally suppressed $(<200 \mathrm{cpm})$.

8000

7000

6000

5000

4000

3000

2000

1000

0
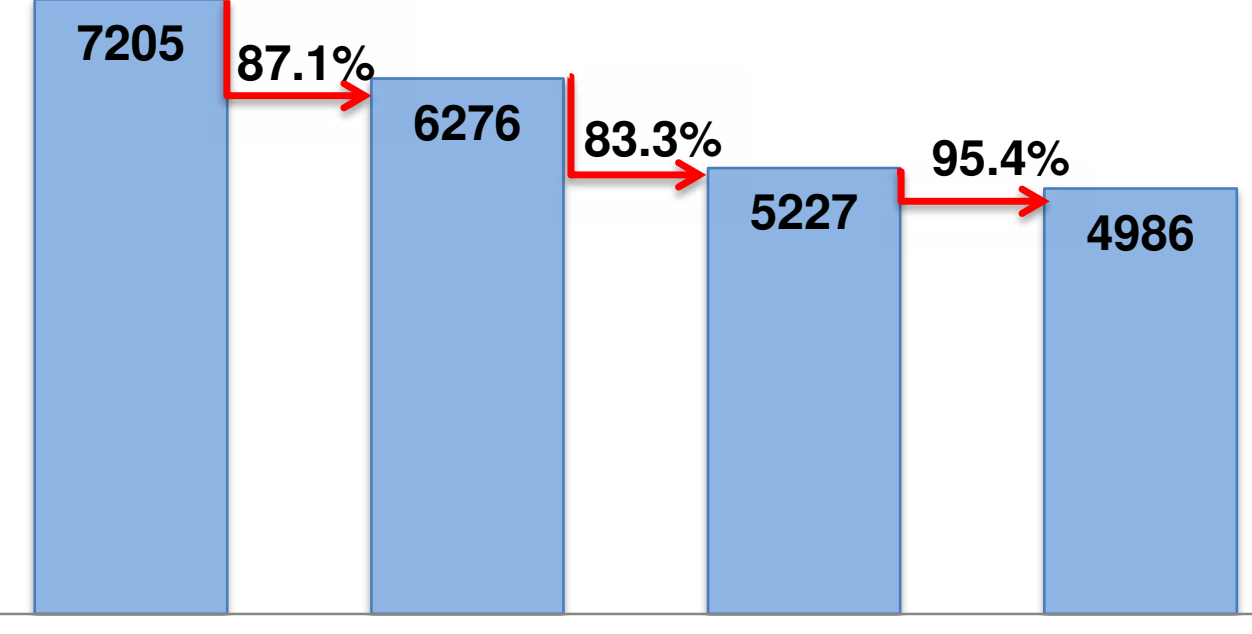

PLHIV*

Diagnosed $^{*}$

On ART^

Virally suppressed ${ }^{\wedge}$ $(<200)$

$\wedge$ obtained by clinical audit

Figure 2. 4-stage continuum of HIV care

\section{Conclusion}

- Monitoring the continuum of HIV care in Ireland is feasible, providing valuable information for action in the HIV response

- Ireland is above the EU/EEA average for the 90-90-90 targets, when compared to the latest complete ECDC report ${ }^{2}$

- Areas for focus include improving engagement and retention in care and reducing the proportion of undiagnosed cases

- Future work will involve further refinement of the modelling and include analysis by individual population groups. 